\title{
Sepsis-induced cardiomyopathy is associated with higher mortality rates in patients with sepsis
}

\author{
Balaram Krishna J Hanumanthu', Anika Sasidharan Nair ${ }^{2}$, Adarsh Katamreddy ${ }^{2}$, Jason S Gilbert ${ }^{3}$, \\ Jee Young You ${ }^{2}$, Obiageli Lynda Offor ${ }^{2}$, Ankit Kushwaha ${ }^{2}$, Ankita Krishnan'2 ${ }^{2}$ Marzio Napolitano², \\ Leonidas Palaidimos ${ }^{2}$, Joaquin Morante ${ }^{4}$, Seema S. Tekwani ${ }^{5}$, Suchita Mehta ${ }^{6}$, Aanchal Gupta ${ }^{7}$, \\ Harmeen Goraya ${ }^{8}$, Mengyang Sun ${ }^{9}$, Robert T. Faillace ${ }^{2}$, Perminder Gulani ${ }^{4}$
}

${ }^{1}$ Department of Cardiology, Mount Sinai Beth Israel Hospital, New York, NY; ${ }^{2}$ Department of Medicine, Jacobi Medical Center, Albert Einstein College of Medicine, New York, NY; ${ }^{3}$ Albert Einstein College of Medicine, Bronx, NY; ${ }^{4}$ Division of Pulmonary and Critical Care Medicine, Department of Medicine, Jacobi Medical Center, Albert Einstein College of Medicine, New York, NY, ${ }^{5}$ Division of Pulmonary, Allergy, Critical Care and Sleep Medicine, Emory University School of Medicine, Atlanta, GA; Institute of Critical Care Medicine, Mount Sinai Hospital, New York, NY; ${ }^{7}$ Division of Pulmonary and Critical Care Medicine, Beth Israel Lahey Health, Burlington, MA; ${ }^{8}$ Division of Pulmonary and Critical Care Medicine, Department of Internal Medicine, University of Arkansas for Medical Sciences Medical Center, Little Rock, AR; ${ }^{9}$ Department of Obstetrics and Gynecology, Jacobi Medical Center, Albert Einstein College of Medicine, New York, NY, USA

Background: Patients with sepsis are at risk for developing sepsis-induced cardiomyopathy (SIC). Previous studies offer inconsistent results regarding the association of SIC and mortality. This study sought to assess whether SIC is linked to mortality in patients with sepsis and to evaluate predictors of the development of SIC.

Methods: In this retrospective study, patients admitted to the medical intensive care unit with a diagnosis of sepsis in the absence of acute coronary syndrome were included. SIC was identified using transthoracic echo and was defined by a new onset decline in left ventricular ejection fraction (LVEF) $\leq 50 \%$, or $\geq 10 \%$ decline in LVEF compared to baseline in patients with a history of heart failure with reduced ejection fraction. Multivariable logistic regression analysis was performed using the $\mathrm{R}$ software program.

Results: Of the 359 patients in the final analysis, 19 (5.3\%) had SIC. Eight (42.1\%) of the 19 patients in the SIC group and $60(17.6 \%)$ of the 340 patients in the non-SIC group died during hospitalization. SIC was associated with an increased risk for all-cause in-hospital mortality (odds ratio [OR], 4.46; 95\% confidence interval [CI], 1.15-18.69; $\mathrm{P}=0.03$ ). Independent predictors for the development of SIC were albumin level $(\mathrm{OR}, 0.47 ; 95 \% \mathrm{Cl}, 0.23-0.93$; $\mathrm{P}=0.03)$ and culture positivity $(\mathrm{OR}, 8.47 ; 95 \% \mathrm{Cl}, 2.24-55.61 ; \mathrm{P}=0.006)$. Concomitant right ventricular hypokinesis was noted in $13(68.4 \%)$ of the 19 SIC patients.

Conclusions: SIC was associated with an increased risk for all-cause in-hospital mortality. Low albumin level and culture positivity were independent predictors of SIC.

Key Words: cardiomyopathy; mortality; sepsis; septic shock

\section{INTRODUCTION}

Sepsis is a dysregulated systemic inflammatory response to an infectious pathogen [1]. While there is now a greater understanding of the immune pathways behind this host response,

\section{Original Article}

Received: February 27, 2021

Revised: May 17, 2021

Accepted: May 18, 2021

Corresponding author

Perminder Gulani

Division of Pulmonary and Critical

Care Medicine, Department of

Medicine, Jacobi Medical Center,

1400 Pelham Parkway South, Bronx, NY 10461, USA

Tel: +1-573-289-5865

Fax: +1-718-918-7460

E-mail: perminder.gulani@nychhc. org

Copyright $(92021$ The Korean Society of Critical Care Medicine

This is an Open Access article distributed under the terms of Creative Attributions Non-Commercial License (https:// creativecommons.org/li-censes/by-nc/4.0/) which permits unrestricted noncommercial use, distribution, and reproduction in any medium, provided the original work is properly cited. 
sepsis remains an elusive syndrome that often leads to poor outcomes [2]. Inherent to the progression of sepsis towards a worsening clinical status is organ dysfunction, and the heart is one such organ that can be injured by the inflammatory response. Myocardial depression in septic patients was first described by Parker et al. [3] in 1984; however, the definition has since evolved to include specific components that characterize this unique cardiomyopathy, including global, biventricular, systolic, and diastolic dysfunction; left ventricle dilation; and reversibility within seven to 10 days [4].

While sepsis-induced cardiomyopathy (SIC) is frequently observed, the impact of SIC on the mortality of patients with sepsis remains unclear. Some studies have suggested that SIC is associated with increased mortality [5], while many others report no difference in mortality among septic patients with or without SIC [6-8]. There are even a few earlier studies that argue SIC is associated with a decrease in mortality [3], suggesting that global cardiac hypokinesis (caused by dilated and failing ventricles in the case of SIC) may protect against the heightened mortality rates that may be seen in concert with the hyperkinetic state of sepsis [9].

In addition to the discrepancies in the literature regarding the association between SIC and mortality, conflicts also exist concerning which patient-specific factors predict the progression to SIC [4]. Recent studies differ in their conclusions of whether factors such as age, diabetes mellitus (DM), pre-existing heart failure, and lactate and cardiac biomarkers predict the progression to SIC $[10,11]$.

This study examined SIC as a prognostic indicator for mortality in patients with sepsis and sought to elucidate which risk factors most strongly portend the development of SIC in septic patients.

\section{MATERIALS AND METHODS}

\section{Study Design}

In this retrospective study, we analyzed charts for all patients admitted with a diagnosis of sepsis between January 1, 2016, and December 31, 2017, to the 12-bed medical intensive care unit of a municipal hospital in New York. Sepsis was defined as life-threatening organ dysfunction caused by a dysregulated host response to infections (indicated by a 2-point increase in the Sequential Organ Failure Assessment [SOFA] score) per the Third International Consensus Definitions for Sepsis and Septic Shock [1]. Infection was defined as the positive detection of microorganisms in culture or as radiological or clinical manifestations suggesting infection despite negative culture

\section{KEYMESSAGES}

- Sepsis-induced cardiomyopathy (SIC) is associated with a higher mortality rate in patients with sepsis, even following adjustment for sepsis severity scores in the final analysis of mortality.

- Factors indicating the severity of sepsis (such as low albumin level) rather than those indicating cardiac risk (e.g., diabetes mellitus, cardiac biomarkers) were associated with the development of SIC.

- Biventricular dysfunction was more common than isolated left ventricular dysfunction in SIC.

results [12]. Septic shock was defined as sepsis with a vasopressor requirement to maintain a mean arterial pressure of greater than $65 \mathrm{~mm} \mathrm{Hg}$ and serum lactate level of greater than $2 \mathrm{mEq} / \mathrm{L}$ in the absence of hypovolemia. Sepsis and septic shock were treated according to the most recent Surviving Sepsis Campaign guidelines [13].

This study was approved by the Institutional Review Board of the institution of Albert Einstein College of Medicine (IRB No. 2018-8773). The requirement for informed consent was waived due to the retrospective nature of this study as well as its design. All authors vouch for the accuracy and completeness of the data and the fidelity of the final study to the research protocol.

\section{Patient Selection}

Charts were reviewed for all adults (aged $\geq 18$ years) admitted to the medical intensive care unit with an initial admission diagnosis of sepsis as assessed by the admitting team. Parameters were reviewed to confirm that the sepsis diagnosis was consistent with the Third International Consensus Definitions for Sepsis and Septic Shock. Patients were included in the study if they had a transthoracic echocardiogram (TTE) performed within 72 hours of admission and a comparison TTE completed either six months prior to admission (baseline TTE) or within three months after the diagnosis of sepsis (follow-up TTE).

Patients were divided into SIC and non-SIC groups. The SIC group included patients with left ventricular ejection fraction (LVEF) values of up to $50 \%$ in the setting of sepsis and evidence of either reversibility (defined as a return to normal LVEF on follow-up TTE) and/or novelty (defined as a decline of $\geq 10 \%$ in LVEF vs. baseline TTE). The non-SIC group included patients with normal LVEF or those with pre-existing reduced LVEF and a decline of less than $10 \%$ in LVEF in the setting of sepsis. All TTEs were read by a board-certified cardiologist. 
Patients were excluded if they met any of the following criteria: history of recent acute coronary syndrome or heart failure exacerbation; primary diagnosis of acute coronary syndrome during the index admission; or moderate to severe mitral or aortic regurgitation, which would preclude accurate LVEF measurements without invasive hemodynamic measures.

\section{Data Collection}

Data collected include patient demographic and baseline characteristics (age, sex, body mass index, tobacco use history, race, and ethnicity), medical history (hypertension, DM, prior symptomatic heart failure, angiographically confirmed coronary artery disease, cirrhosis, atrial fibrillation, human immunodeficiency virus infection, prior cerebrovascular disease, active or prior cocaine and/or ethanol use), medication use (betablockers, angiotensin-converting enzyme inhibitors, angiotensin receptor blockers, mineralocorticoid receptor antagonists, anticoagulants), clinical characteristics (Glasgow coma scale at the time of diagnosis of sepsis, blood pressure, heart rate, respiratory rate, temperature, need for vasopressors, mechanical ventilation, and/or renal replacement therapy), laboratory characteristics (complete blood count, electrolytes, renal and hepatic functions, arterial blood gases, coagulation profile, troponin T, lactate, and microbiological data), and echocardiographic data (function and size of the left and right ventricles [LV and RV, respectively]). Culture positivity was defined as the growth of at least one microorganism in body fluids (urine, blood, sputum, bronchoalveolar lavage, cerebrospinal fluid, or peritoneal fluid) and/or biopsy specimens. These variables were used to calculate the Acute Physiology And Chronic Health Evaluation (APACHE) II score at the time of admission and SOFA score at the time of admission and at 48 hours, respectively. The desired data were extracted from electronic health records manually and stored in a password-protected database for analysis.

\section{Statistical Analysis}

Baseline characteristics were analyzed using descriptive statistics, including mean and standard deviation values for continuous parametric variables, median and interquartile range values for nonparametric data, and frequency (percentage) values for categorical or nominal variables. Baseline continuous variables were compared between the two groups using a t-test or nonparametric equivalent. The chi-square test was employed for the comparison of categorical/nominal variables. Multivariable logistic regression analysis was performed to determine the association of SIC with all-cause in-hospital mortality. Statistical significance was set at $\mathrm{P}<0.05$. Data were analyzed using a dedicated statistical analysis software program (R ver. 4.0.2; R Foundation for Statistical Computing, Vienna, Austria).

\section{RESULTS}

A total of 359 patients were included in the analysis, of whom

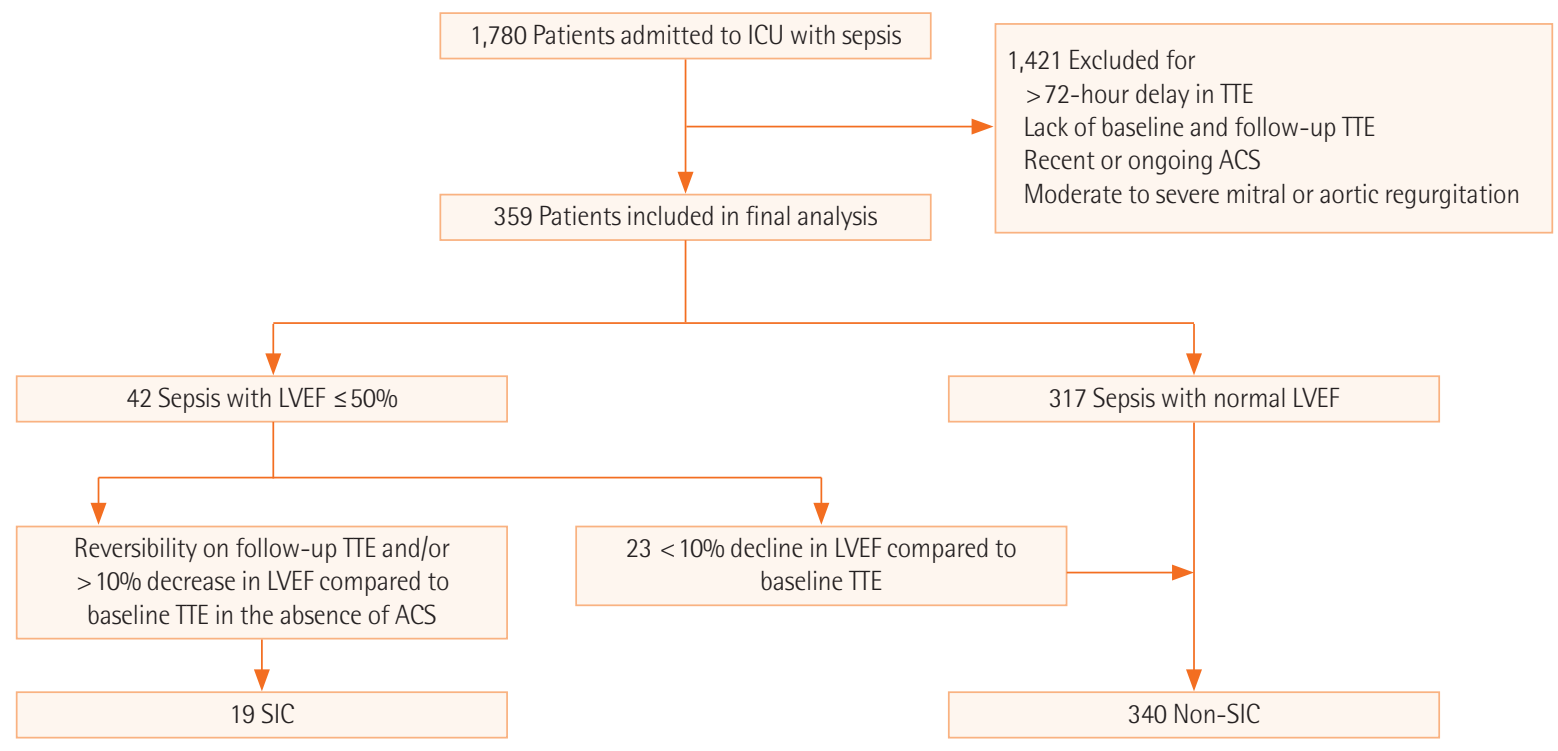

Figure 1. Patient flowchart. ICU: intensive care unit; TTE: transthoracic echocardiogram; ACS: acute coronary syndrome; LVEF: left ventricular ejection fraction; SIC: sepsis-induced cardiomyopathy. 
Table 1. Baseline patient characteristics

\begin{tabular}{|c|c|c|c|}
\hline Variable & Non-SIC $(n=340)$ & $\operatorname{SIC}(n=19)$ & P-value ${ }^{a}$ \\
\hline Age (yr) & $67 \pm 16$ & $64 \pm 18$ & 0.412 \\
\hline Female sex & $185(54.4)$ & $12(63.2)$ & 0.541 \\
\hline Diabetes mellitus & $137(40.2)$ & $11(57.9)$ & 0.101 \\
\hline Hypertension & $224(65.9)$ & $12(63.2)$ & 0.803 \\
\hline CAD & $58(17.1)$ & $4(21.1)$ & 0.734 \\
\hline History of heart failure & $59(17.4)$ & $7(36.8)$ & 0.029 \\
\hline ESRD & $28(8.2)$ & $1(5.3)$ & 0.591 \\
\hline History of CVA & $78(22.9)$ & $3(15.8)$ & 0.502 \\
\hline COPD & $73(21.5)$ & $5(26.3)$ & 0.613 \\
\hline Cirrhosis & $31(9.1)$ & $2(10.5)$ & 0.804 \\
\hline Beta-blocker use & $128(38.0)$ & $9(47.4)$ & 0.565 \\
\hline APACHE II score & $19(13.0-24.2)$ & $22(18.5-27.5)$ & 0.016 \\
\hline Lactate (mmol/L) & $3.2(1.8-6.4)$ & $4.6(3.0-8.3)$ & 0.108 \\
\hline Troponin T ( $\mu \mathrm{g} / \mathrm{L})$ & $0.1(0.0-0.4)$ & $0.2(0.0-1.1)$ & 0.186 \\
\hline Albumin (g/dl) & $3.23 \pm 0.76$ & $2.57 \pm 0.63$ & $<0.001$ \\
\hline \multicolumn{4}{|l|}{ Echocardiographic parameter } \\
\hline LVEF & $64.0(58.0-70.0)$ & $30.0(20.0-37.5)$ & $<0.001$ \\
\hline Regional wall motion abnormality & $30(9.0)$ & $9(50.0)$ & $<0.001$ \\
\hline RV dilation & $23(7.1)$ & $2(13.3)$ & 0.304 \\
\hline RV hypokinesis & $25(7.4)$ & $13(68.4)$ & $<0.001$ \\
\hline Shock requiring vasopressors & $128(37.6)$ & $16(84.2)$ & $<0.001$ \\
\hline Mechanical ventilation & $151(44.4)$ & 17 (89.5) & $<0.001$ \\
\hline Culture positivity & $140(41.1)$ & $17(89.5)$ & $<0.001$ \\
\hline Mortality & $60(17.6)$ & $8(42.1)$ & 0.008 \\
\hline
\end{tabular}

Values are presented as mean \pm standard deviation, number (\%), or median (interquartile range).

SIC: sepsis-induced cardiomyopathy; CAD: coronary artery disease; ESRD: end-stage renal disease; CVA: cerebrovascular accident/transient ischemic accident; COPD: chronic obstructive pulmonary disease; APACHE: Acute Physiology and Chronic Health Evaluation; LVEF: left ventricular ejection fraction; RV: right ventricular.

aP-value for chi-square test (categorial variables: female, diabetes mellitus, hypertension, CAD, history of heart failure, ESRD, CVA, COPD, cirrhosis, regional wall motion abnormality, RV dilation, RV hypokinesis, shock requiring vasopressors, mechanical ventilation, culture positivity, and mortality), ttest (continuous variables: age and albumin), or Kruskal-Wallis test (skewed distribution: lactate, troponin T, and LVEF); significant values P $<0.05$; ${ }^{\circ} \mathrm{Growth}$ of at least one microorganism in body fluids and/or biopsy specimens.

19 were found to have SIC. Of the 340 patients in the control group, 23 had a history of heart failure with reduced $\mathrm{EF}$ and their LVEF was decreased to less than $10 \%$ relative to as determined by baseline TTE (Figure 1). Baseline characteristics of the study participants are summarized in Table 1. Sixteen (84.2\%) of the 19 patients in the SIC group and 128 (37.6\%) of the 340 patients in the non-SIC group met the definition of septic shock and required vasopressors. Seventeen (89.5\%) of the patients in the SIC group and 151 (44.4\%) of the patients in the non-SIC group required mechanical ventilation. Finally, eight (42.1\%) of the patients in the SIC group and 60 (17.6\%) of the patients in non-SIC group died during hospitalization.
Reversibility demonstrating recovery of LVEF to normal was confirmed by follow-up TTE in 10 of the 11 patients who survived in the SIC group. Concomitant right ventricular (RV) hypokinesis was noted in 13 (68.4\%) of the SIC patients.

Amongst the 359 study participants, the following seven factors were found to be associated with increased all-cause in-hospital mortality in univariable unadjusted analysis: SIC (odds ratio [OR], 4.35; 95\% confidence interval [CI], 2.19-8.63; $\mathrm{P}<0.01$ ), albumin level (OR, 0.41; 95\% CI, 0.29-0.57; $\mathrm{P}<0.01$ ), APACHE II (OR, 1.77; 95\% CI, 1.04-2.27; $\mathrm{P}<0.001)$, culture positivity (OR, 1.81; 95\% CI, 1.10-3.01; $\mathrm{P}=0.02)$, lactate (OR, 1.26; 95\% CI, 1.18-1.35; $\mathrm{P}<0.001)$, shock requiring vasopres- 
Table 2. Predictors of mortality in patients admitted to the medical intensive care unit with sepsis

\begin{tabular}{|c|c|c|c|c|}
\hline \multirow{2}{*}{ Variable } & \multicolumn{2}{|c|}{ Univariable model } & \multicolumn{2}{|c|}{ Multivariable model } \\
\hline & $\mathrm{OR}(95 \% \mathrm{Cl})$ & P-value ${ }^{a}$ & OR $(95 \% \mathrm{Cl})$ & P-value ${ }^{a}$ \\
\hline Sepsis-induced cardiomyopathy & $4.35(2.19-8.63)$ & $<0.001$ & $4.46(1.15-18.69)$ & 0.031 \\
\hline Albumin level $^{b}$ & $0.41(0.29-0.57)$ & $<0.001$ & $0.63(0.40-0.99)$ & 0.048 \\
\hline APACHE II score ${ }^{c}$ & $1.77(1.04-2.27)$ & $<0.001$ & $1.11(0.80-1.58)$ & 0.503 \\
\hline Culture positivity $^{d}$ & $1.81(1.10-3.01)$ & 0.022 & $0.84(0.42-1.64)$ & 0.612 \\
\hline Lactate $^{e}$ & $1.26(1.18-1.35)$ & $<0.001$ & $1.12(1.13-1.31)$ & $<0.001$ \\
\hline Shock requiring vasopressors & $7.03(4.05-12.60)$ & $<0.001$ & $1.76(0.83-3.77)$ & 0.131 \\
\hline Mechanical ventilation & $9.18(4.98-18.15)$ & $<0.001$ & $3.36(1.52-7.77)$ & 0.003 \\
\hline History of heart failure & $1.29(0.68-2.36)$ & 0.407 & & \\
\hline $\operatorname{Sex}^{f}$ & $1.09(0.66-1.80)$ & 0.728 & & \\
\hline $\operatorname{Age}^{g}$ & $1.00(0.99-1.02)$ & 0.333 & & \\
\hline CAD & $1.04(0.52-1.97)$ & 0.891 & & \\
\hline ESRD & $1.99(0.85-4.39)$ & 0.092 & & \\
\hline HIV & $0.76(0.21-2.10)$ & 0.631 & & \\
\hline Troponin $T^{h}$ & $1.00(0.97-1.02)$ & 0.844 & & \\
\hline Beta-blocker use & $0.66(0.38-1.11)$ & 0.132 & & \\
\hline History of CVA & $0.84(0.35-1.83)$ & 0.691 & & \\
\hline COPD & $1.23(0.55-2.57)$ & 0.591 & & \\
\hline Cirrhosis & $0.78(0.18-2.34)$ & 0.693 & & \\
\hline Hypertension & $0.82(0.49-1.39)$ & 0.454 & & \\
\hline Diabetes mellitus & $1.02(0.61-1.70)$ & 0.912 & & \\
\hline
\end{tabular}

OR: odds ratio; Cl: confidence interval; APACHE: Acute Physiology And Chronic Health Evaluation; CAD: coronary artery disease; ESRD: end-stage renal disease; HIV: human immunodeficiency virus; CVA: cerebrovascular accident; COPD: chronic obstructive pulmonary disease.

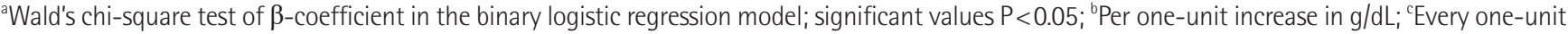
increase in APACHE II score; ${ }^{\circledR}$ Growth of at least one microorganism in body fluids and/or biopsy specimens; ' ${ }^{2}$ actate per one-unit increase in mmol/L;

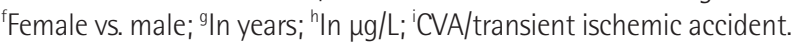

sors (OR, 7.03; 95\% CI, 4.05-12.60; $\mathrm{P}<0.001)$, and mechanical ventilation (OR, 9.18; 95\% CI, 4.98-18.15; $\mathrm{P}<0.001$ ) (Table 2). In the multivariable regression model analysis, after adjusting for albumin level, APACHE II score, culture positivity, lactate, shock requiring vasopressors, and mechanical ventilation, SIC was associated with increased all-cause in-hospital mortality (OR, 4.46; 95\% CI, 1.15-18.69; $\mathrm{P}=0.03$ ) (Table 2).

Albumin level and culture positivity were independently associated with the development of SIC in the multivariable regression model (Table 3). Each one-unit increase in the albumin level ( $\mathrm{g} / \mathrm{dl}$ ) was associated with a lower likelihood of developing SIC (OR, 0.47; CI, 0.23-0.93; $\mathrm{P}=0.03$ ). Meanwhile, culture positivity was associated with a greater likelihood of developing SIC (OR, 8.47; CI, 2.24-55.61; P=0.006). In our cohort, age, history of DM, and cardiac biomarkers were not associated with a greater likelihood of SIC.

\section{DISCUSSION}

Our study evaluated the predictors of SIC and the association of SIC with mortality in sepsis. Our study demonstrates as association between SIC and increased mortality in patients with sepsis, and this association was maintained after adjusting for sepsis severity indices. We also found that low albumin and culture positivity were independently associated with development of SIC. Additionally, we noted biventricular dysfunction was more common than isolated left ventricular (LV) dysfunction in SIC. Lastly, the incidence of SIC was $5.2 \%$ among our cohort of patients admitted to the ICU with sepsis.

Prior to 2010, studies on SIC showed that, during sepsis, patients who developed reduced EF were more likely to survive relative to those who continued to have normal or hyperdynamic LV systolic function [3,9,14-16]. This contrasts with our finding of an association between SIC and greater mortality 
Table 3. Predictors of SIC in patients admitted to the medical intensive care unit with sepsis

\begin{tabular}{|c|c|c|c|c|}
\hline \multirow{2}{*}{ Variable } & \multicolumn{2}{|c|}{ Univariable model } & \multicolumn{2}{|c|}{ Multivariable model } \\
\hline & OR $(95 \% \mathrm{Cl})$ & P-value ${ }^{a}$ & OR $(95 \% \mathrm{Cl})$ & P-value ${ }^{a}$ \\
\hline Albumin level $^{b}$ & $0.55(0.36-0.84)$ & 0.006 & $0.47(0.23-0.93)$ & 0.031 \\
\hline History of heart failure & $3.15(1.53-6.34)$ & 0.001 & $2.72(0.87-8.10)$ & 0.072 \\
\hline APACHE II score ${ }^{c}$ & $1.53(1.13-2.09)$ & 0.007 & $1.10(0.66-1.88)$ & 0.714 \\
\hline Culture positivity $^{d}$ & $3.47(1.74-7.32)$ & $<0.001$ & $8.47(2.24-55.61)$ & 0.006 \\
\hline Shock requiring vasopressors & $8.91(2.90-38.87)$ & $<0.001$ & $2.23(0.57-11.39)$ & 0.271 \\
\hline Mechanical ventilation & $10.75(3.02-68.48)$ & 0.002 & $4.38(0.95-32.08)$ & 0.081 \\
\hline $\operatorname{Sex}^{\mathrm{e}}$ & $1.01(0.52-1.99)$ & 0.954 & & \\
\hline $\operatorname{Age}^{f}$ & $1.01(0.99-1.03)$ & 0.212 & & \\
\hline CAD & $2.00(0.90-4.16)$ & 0.073 & & \\
\hline ESRD & $1.75(0.56-4.56)$ & 0.284 & & \\
\hline HIV & $1.90(0.53-5.45)$ & 0.271 & & \\
\hline Lactate $^{g}$ & $1.04(0.98-1.11)$ & 0.152 & & \\
\hline Troponin $T^{h}$ & $0.98(0.87-1.01)$ & 0.644 & & \\
\hline Beta-blocker use & $1.51(0.77-2.93)$ & 0.221 & & \\
\hline Diabetes mellitus & $2.03(0.80-5.39)$ & 0.143 & & \\
\hline Hypertension & $0.89(0.35-2.46)$ & 0.821 & & \\
\hline
\end{tabular}

SIC: sepsis-induced cardiomyopathy; OR: odds ratio; Cl: confidence interval; APACHE: Acute Physiology and Chronic Health Evaluation; CAD: coronary artery disease; ESRD: end-stage renal disease; HIV: human immunodeficiency virus.

${ }^{a} \mathrm{P}$-value for Wald's chi-square test of $\beta$-coefficient in the binary logistic regression model; significant values $\mathrm{P}<0.05$; ${ }^{\text {bPer }}$ one-unit increase in g/dl;

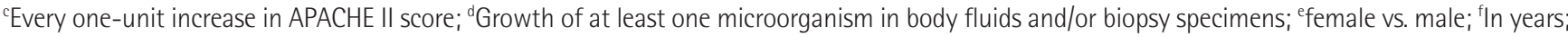
${ }^{9}$ Per one-unit increase in $\mathrm{mmol} / \mathrm{L} ;{ }^{\mathrm{h}} \mathrm{In} \mu \mathrm{g} / \mathrm{L}$.

among patients with sepsis. Of course, some possible explanations may account for these differences. First, unlike in our study, the aforementioned previous studies did not adjust for sepsis severity scores in the final analysis of mortality. Second, the first-line vasopressor used in our study was norepinephrine, based on the current sepsis guidelines, whereas earlier studies employed dopamine or epinephrine [3,9,14], which are potentially harmful in a hyperdynamic state, given their higher beta-1 agonist activity.

Biventricular dysfunction was more common than isolated LV dysfunction in patients with SIC. RV hypokinesis, a marker of RV dysfunction, was present in $68 \%$ of our SIC patients. This is likely an underestimation of the rate of RV dysfunction. Achieving an accurate estimation of RV dysfunction is challenging as reliable and clinically useful markers for RV function are still under active investigation [17]. Other investigators have previously demonstrated the potential for biventricular dysfunction in SIC, and RV dysfunction rates of up to $52 \%$ have been reported in the setting of septic shock [18].

The incidence of SIC was $5.2 \%$ in our study evaluating patients with sepsis. This is comparable to rates in other studies that did not limit the study population to only those with shock $[6,19]$. However, an incidence of up to $60 \%$ has been reported by one study focusing on patients with septic shock using daily transesophageal echocardiogram (TEE) [14]. Apart from the aforementioned disparity in patient populations, differences in technique (TEE vs. TTE) and frequency of imaging (daily TEE vs. one-time TTE) may account for the variation in incidence. Our study, along with prior investigations, showed that cardiomyopathy is a common phenomenon in sepsis and that its likelihood increases in patients who progress to shock.

We found that both low albumin level and culture positivity were independently associated with the development of SIC. Albumin, a negative acute-phase reactant, decreases with worsening sepsis and is an indicator of the severity of sepsis [20]. Culture positivity, while it does not directly predict the severity of sepsis, could be representative of the burden of infection [21]. Other factors, such as APACHE II score, mechanical ventilation, and shock requiring vasopressors [22] — which are all indicators of sepsis severity-were found to be significant predictors of SIC in the univariable analysis; however, in the multivariable analysis, the P-values trended towards statistical significance but ultimately did not reach the cutoff value $(\mathrm{P}<0.05)$. 
We believe that the lack of significance was due to the small sample size and inadequate power of this study. We hypothesize that, in a larger study, APACHE II score, mechanical ventilation, shock requiring vasopressors, and a history of heart failure would be independently associated with the development of SIC. Some previously reported factors, such as age and DM, were not identified as predictors of SIC in our cohort. Elevated troponin was also not associated with the development of SIC, consistent with findings from prior research [12]. These results suggest that the severity of sepsis is a stronger predictor of SIC than cardiac risk factors. Thus, factors indicating the severity of sepsis (e.g., low albumin level, APACHE II score) would be more strongly associated with the development of SIC than those indicating cardiac risk (e.g., DM, cardiac biomarkers).

Although our study has a number of novel findings, they need to be interpreted in the context of the following limitations. First, we may have underestimated LV systolic dysfunction by using LVEF as a marker. Using LV end-systolic elastance (LVESE) or global longitudinal strain values may offer a more accurate estimate of LV systolic dysfunction, as these measures do not vary with preload or afterload, both of which are dynamic in the setting of sepsis. LVESE was not assessed in our study and is not routinely assessed clinically. Second, as noted earlier in the discussion, RV dysfunction may also have been underestimated since we used RV hypokinesis as the clinical surrogate. Third, SIC is rapidly reversible over the course of days. Since we only included patients who underwent TTE within 72 hours of diagnosis of sepsis in our study, the incidence of SIC may actually be higher or lower depending on the timing of imaging. Fourth, we employed a multivariable Cox proportional hazards model for analysis and, importantly, two inherent limitations of this method of statistical analysis exist, including that the effect of unknown confounders cannot be accounted for in the study outcome and, due to the small sample size in the SIC group, the possibility of overfitting cannot be excluded.

SIC is a complex reversible cardiomyopathy seen in patients with sepsis and septic shock. Data regarding mortality in SIC have been conflicting. Our study demonstrated that SIC was an independent predictor of mortality in septic patients. Biventricular dysfunction was more common than LV dysfunction alone in patients with SIC. We found that culture positivity and low albumin were independent predictors for the development of SIC, while DM, a history of heart failure, and cardiac biomarkers were not.

\section{CONFLICT OF INTEREST}

No potential conflict of interest relevant to this article was reported.

\section{ORCID}

Balaram Krishna J Hanumanthu https://orcid.org/0000-0003-2637-9736

Anika Sasidharan Nair

https://orcid.org/0000-0003-0458-6574

Adarsh Katamreddy https://orcid.org/0000-0002-1960-7123 Jason S Gilbert https://orcid.org/0000-0002-6395-9054 Jee Young You https://orcid.org/0000-0002-0331-879X Obiageli Lynda Offor https://orcid.org/0000-0002-0977-5668 Ankit Kushwaha https://orcid.org/0000-0002-3678-8739 Ankita Krishnan https://orcid.org/0000-0003-1824-6482 Marzio Napolitano https://orcid.org/0000-0002-6855-4072 Leonidas Palaidimos https://orcid.org/0000-0003-4682-5991 Joaquin Morante https://orcid.org/0000-0002-0354-2839 Seema S. Tekwani https://orcid.org/0000-0002-7395-1160 Suchita Mehta https://orcid.org/0000-0001-5009-3768 Aanchal Gupta Harmeen Goraya Mengyang Sun Robert T. Faillace https://orcid.org/0000-0002-8033-7302 https://orcid.org/0000-0001-9149-8868 https://orcid.org/0000-0002-3998-8744 https://orcid.org/0000-0002-9149-7862

Perminder Gulani

\section{AUTHOR CONTRIBUTIONS}

Conceptualization: BKJH, RTF, PG. Methodology: BKJH, AK (Adarsh Katamreddy), JSG, JYY, RTF, PG. Formal analysis: BKJH, ASN, AK (Adarsh Katamreddy), JYY, PG. Data curation: BKJH, ASN, OLO, AK (Ankit Kushwaha), AK (Ankita Krishnan), MN. Project administration: BKJH, LP, JM, ST, SM, AG, HG, MS, RTF, PG. Visualization: BKJH, PG. Writing - Original draft: ASN, AK (Adarsh Katamreddy), JSG, JYY, MS, PG. Writing - Review and editing: BKJH, ASN, AK (Adarsh Katamreddy), OLO, AK (Ankit Kushwaha), AK (Ankita Krishnan), MN, LP, JM, ST, SM, AG, HG, MS, RTF, PG. Writing-review \& editing: all authors.

\section{REFERENCES}

1. Singer M, Deutschman CS, Seymour CW, Shankar-Hari M, Annane D, Bauer M, et al. The third international consensus definitions for sepsis and septic shock (Sepsis-3). JAMA 2016; 
315:801-10.

2. Angus DC, van der Poll T. Severe sepsis and septic shock. N Engl J Med 2013;369:840-51.

3. Parker MM, Shelhamer JH, Bacharach SL, Green MV, Natanson C, Frederick TM, et al. Profound but reversible myocardial depression in patients with septic shock. Ann Intern Med 1984;100:483-90.

4. L'Heureux M, Sternberg M, Brath L, Turlington J, Kashiouris MG. Sepsis-induced cardiomyopathy: a comprehensive review. Curr Cardiol Rep 2020;22:35.

5. Landesberg G, Gilon D, Meroz Y, Georgieva M, Levin PD, Goodman S, et al. Diastolic dysfunction and mortality in severe sepsis and septic shock. Eur Heart J 2012;33:895-903.

6. McLean AS, Huang SJ, Hyams S, Poh G, Nalos M, Pandit R, et al. Prognostic values of B-type natriuretic peptide in severe sepsis and septic shock. Crit Care Med 2007;35:1019-26.

7. Furian T, Aguiar C, Prado K, Ribeiro RV, Becker L, Martinelli $\mathrm{N}$, et al. Ventricular dysfunction and dilation in severe sepsis and septic shock: relation to endothelial function and mortality. J Crit Care 2012;27:319.e9-15.

8. Sturgess DJ, Marwick TH, Joyce C, Jenkins C, Jones M, Masci $\mathrm{P}$, et al. Prediction of hospital outcome in septic shock: a prospective comparison of tissue Doppler and cardiac biomarkers. Crit Care 2010;14:R44.

9. Vieillard Baron A, Schmitt JM, Beauchet A, Augarde R, Prin S, Page B, et al. Early preload adaptation in septic shock? A transesophageal echocardiographic study. Anesthesiology 2001; 94:400-6.

10. Sato R, Kuriyama A, Takada T, Nasu M, Luthe SK. Prevalence and risk factors of sepsis-induced cardiomyopathy: a retrospective cohort study. Medicine (Baltimore) 2016;95:e5031.

11. Jeong HS, Lee TH, Bang CH, Kim JH, Hong SJ. Risk factors and outcomes of sepsis-induced myocardial dysfunction and stressinduced cardiomyopathy in sepsis or septic shock: a comparative retrospective study. Medicine (Baltimore) 2018;97:e0263.

12. Song MJ, Lee SH, Leem AY, Kim SY, Chung KS, Kim EY, et al. Predictors and outcomes of sepsis-induced cardiomyopathy in critically ill patients. Acute Crit Care 2020;35:67-76.

13. Society of Critical Care Medicine; European Society of Inten- sive Care Medicine. Surviving Sepsis Campaign [Internet]. Prospect (IL): Mount Society of Critical Care Medicine; 2012 [cited 2021 Jul 1]. Available from: https://www.sccm.org/SurvivingSepsisCampaign/Guidelines/Adult-Patients.

14. Jardin F, Fourme T, Page B, Loubières Y, Vieillard-Baron A, Beauchet A, et al. Persistent preload defect in severe sepsis despite fluid loading: a longitudinal echocardiographic study in patients with septic shock. Chest 1999;116:1354-9.

15. Vieillard-Baron A, Caille V, Charron C, Belliard G, Page B, Jardin F. Actual incidence of global left ventricular hypokinesia in adult septic shock. Crit Care Med 2008;36:1701-6.

16. Etchecopar-Chevreuil C, François B, Clavel M, Pichon N, Gastinne H, Vignon P. Cardiac morphological and functional changes during early septic shock: a transesophageal echocardiographic study. Intensive Care Med 2008;34:250-6.

17. Haddad F, Hunt SA, Rosenthal DN, Murphy DJ. Right ventricular function in cardiovascular disease, part I: anatomy, physiology, aging, and functional assessment of the right ventricle. Circulation 2008;117:1436-48.

18. Kimchi A, Ellrodt AG, Berman DS, Riedinger MS, Swan HJ, Murata GH. Right ventricular performance in septic shock: a combined radionuclide and hemodynamic study. J Am Coll Cardiol 1984;4:945-51.

19. Pulido JN, Afessa B, Masaki M, Yuasa T, Gillespie S, Herasevich V, et al. Clinical spectrum, frequency, and significance of myocardial dysfunction in severe sepsis and septic shock. Mayo Clin Proc 2012;87:620-8.

20. Qian SZ, Jin D, Chen ZB, Ye YC, Xiang WW, Ye LM, et al. Hypoalbuminemia, a novel prognostic factor for prediction of long-term outcomes in critically ill patients with septic shock. Int J Clin Exp Med 2019;12:7401-9.

21. Phua J, Ngerng W, See K, Tay C, Kiong T, Lim H, et al. Characteristics and outcomes of culture-negative versus culture-positive severe sepsis. Crit Care 2013;17:R202.

22. Giamarellos-Bourboulis EJ, Norrby-Teglund A, Mylona V, Savva A, Tsangaris I, Dimopoulou I, et al. Risk assessment in sepsis: a new prognostication rule by APACHE II score and serum soluble urokinase plasminogen activator receptor. Crit Care 2012;16:R149. 